\title{
Research Paper \\ The Effect of Eight Weeks of Selected Exercises in Water on Pain and Balance of Female Nurses With Chronic Back Pain
}

\author{
*Havar Ezadi ${ }^{1}\left[\right.$, Narmin Ghanizadeh Hesar ${ }^{2}$ ( \\ 1. Department of Sport Injuries and Corrective Exercises, Faculty of physical Education, Urmia Branch, Islamic Azad University, Urmia, Iran \\ 2. Department of Sport Injuries and Corrective Exercises, Faculty of physical Education, Urmia University, Urmia, Iran.
}

\begin{tabular}{l|l}
$\begin{array}{c}\text { Use yourdevict to san } \\
\text { and read the article online }\end{array}$ & $\begin{array}{l}\text { Citation: Ezadi H, Ghanizadeh Hesar N. [The Effect of Eight Weeks of Selected Exercises in Water on Pain and Balance of } \\
\text { Female Nurses With Chronic Back Pain (Persian)]. Journal of Sport Biomechanics. 2021; 7(1):44-55. https://doi.org/10.32598/ } \\
\text { biomechanics.7.1.3 }\end{array}$ \\
del https://doi.org/10.32598/biomechanics.7.1.3
\end{tabular}

\section{(c) (i) (8)}

Article Info:

Received: 16 Feb 2021

Accepted: 19 Apr 202

Available Online: 01 Jun 2021

Keywords:

Water therapy, Pain,

Balance, Back pain,

Nurse

\section{ABSTRACT}

Objective The purpose of this study was to investigate the effect of eight weeks of selected exercises in water on pain and balance of nurses with chronic back pain.

Methods This semi-experimental study was carried out on 30 nurses working in Sanandaj city hospitals. Selected exercises in water for eight weeks, three sessions per week, which was carried out progressively and with the practice of central stability exercises on the experimental group. To assess the pain and balance of the patients in the pre and post test tests, both groups used the standard questionnaire of Quebec, FBT test, berg balance test. Independent and dependent t-test was used to analyze the data. The significance level was considered as $\mathrm{P}<0.05$.

Results There was a significant difference between the mean scores of pain and the static and dynamic balance in the experimental and control groups, respectively, with a significant decrease and significant increase in the pain $(\mathrm{P}=0.001)$ and static $(\mathrm{P}=0.001)$ and dynamic $(\mathrm{P}=0.001)$ balance variables in the experimental group.

Conclusion Regarding the effectiveness of selected exercises in water on the pain and balance of nurses with non-specific chronic low back pain, it is recommended to design the training protocols for patients with Special attention should be given to the chronic pain, to the practice of water therapy (and to the importance of taking core stability of the workout in these exercises).

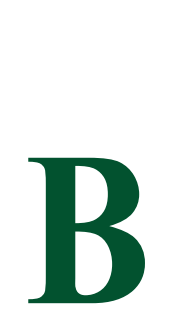

\section{Extended Abstract}

\section{Introduction}

ack pain is one of the most common problems in developing and developed countries that people face problems such as pain and imbalance in daily life. Nurses are considered as high risk groups for low back pain due to their professional work $[1,2]$. The aim of this study was to investigate the effect of eight weeks of select- ed exercises in water on pain and balance of female nurses with chronic low back pain.

\section{Methods}

This quasi-experimental study was performed on 30 nurses working in Sanandaj city hospitals. The selected exercises in water were performed by the experimental group for eight weeks, three sessions per week, which were progressive and with the approach of central stability exercises. To evaluate the pre-test and post-test of pain and balance of

\section{* Corresponding Author:}

Havar Ezadi

Address: Department of Sport Injuries and Corrective Exercises, Faculty of physical Education, Urmia Branch, Islamic Azad University, Urmia, Iran.

Tel: +98 (918) 8744234

E-mail: izadihawar@gmail.com 
the subjects, the standard Quebec questionnaire (for pain assessment), Stork Test (static balance) and LEAF Test (dynamic balance) were used [3, 4]. Independent and dependent t-tests were used to analyze the data. Significance level was considered $\mathrm{P}<0.05$.

\section{Results}

The mean scores of pain and static and dynamic balance were significantly different in the experimental and control groups, so that a significant decrease was observed in pain scores $(\mathrm{P}=0.001)$. There was a significant increase in static balance $(\mathrm{P}=0.001)$ and dynamic balance $(\mathrm{P}=0.001)$ scores, while none of these variables were significant for the control group $(\mathrm{P} \geq 0.05)$.

According to the results of independent t-test in Table 1, there is no significant difference in the pre-test of the control and experimental groups in the variables of pain intensity, static balance and dynamic balance, while the differences in the post-test of these variables are significant $(\mathrm{P} \leq 0.05)$.

\section{Discussion and Conclusion}

The results of this study revealed that selected exercises in water improved pain and balance in the experimental group with non-specific chronic low back pain. Due to the effectiveness of these exercises, it is recommended to pay special attention to hydrotherapy exercises (with the approach of strengthening the central muscles) in designing training protocols for patients with chronic back pain. Regarding the possible mechanism of pain relief for nursing women with chronic non-specific low back pain following the selected water exercise program in this study, it should be noted that the water exercise protocol includes a reduction in weight- bearing forces. Patients who exercise in the water feel lighter and easier to move.

The feeling of losing weight in water seems to relieve or significantly reduce muscle cramps, which in turn reduces muscle spasms. Baker believes that non-weight-bearing exercises are a good option for reducing back pain, and that a person can control his or her weight if he or she is submerged in water [14]. On the other hand, in this study, central stability exercises were used as selected exercises in water. Many researchers consider the role of muscles and soft tissues around the spine in causing low back pain [15]. Their argument is that muscle weakness to support inactive structures against overload may destroy these pain-sensitive structures and ultimately cause pain in these individuals [29].

Previous studies have concluded that abnormalities and weaknesses in both the global and local muscular systems contribute to back pain. Researchers have linked these weaknesses to inappropriate postures that people show during various activities. In most cases, weakness and atrophy are observed in the internal parts of the multifidus muscle and other deep muscles of the lower back, and the relationship between back pain and atrophy of these muscles has been proven in many other studies [29]. Regarding the possible mechanism of improving the balance of female patients with chronic low back pain in this study, it can be stated that in people with low back pain, physiological mechanisms in the balance systems are altered and incorrect information about the spatial position of the body is sent to the brainstem [20]. The higher density of water than air helps the resistance force engage the muscles and increase their strength, which in turn improves patients' balance.

Table 1. Results of independent t-test to compare scores of pain intensity, static and dynamic balance in pre-test and post-test

\begin{tabular}{|c|c|c|c|c|c|c|c|}
\hline Variables & Groups & $\begin{array}{l}\text { Pre-test } \\
\text { Mean Diff. }\end{array}$ & $t$ & $\mathbf{P}$ & $\begin{array}{l}\text { Post-test } \\
\text { Mean Diff. }\end{array}$ & $T$ & $\mathbf{P}$ \\
\hline \multicolumn{8}{|l|}{ Intensity of pain } \\
\hline & Experimental & & & & & & \\
\hline \multirow{2}{*}{$\begin{array}{c}\text { Static balance } \\
\text { Stork test (seconds) }\end{array}$} & Control & 0.88 & 0.45 & 0.76 & -16.09 & -8.13 & 0.001 \\
\hline & Experimental & & & & & & \\
\hline \multirow{2}{*}{$\begin{array}{l}\text { Dynamic balance } \\
\text { LEAF test (score) }\end{array}$} & Control & 0.80 & 0.66 & 0.51 & -11.35 & -9.45 & 0.001 \\
\hline & Experimental & & & & & & \\
\hline
\end{tabular}


In this study, other causes of improved balance can be attributed to the physical properties of water. Immersion of the body and floating in the water environment increases the input of deep receptors and improves the balance by more adjustment and stability of the body. On the other hand, because water has a higher viscosity than air, it has a higher resistance. Therefore, sensory feedback in the water environment increases, and thus improves the body's sense of awareness. Other effects of exercise in water on improving balance include stimulating the atrial system and facilitating atrial inlets $[22,23]$. Exposure to water can enhance the stimulation of the skin inlets and thus increase the stimulation of the afferent nerves. Therefore, multisensory exercises such as water exercises may stimulate the senses involved in balance to provide conditions for challenging the balance system, and thus be effective in improving balance [15].

\section{Ethical Considerations}

Compliance with ethical guidelines

All ethical principles are considered in this article. The participants were informed about the purpose of the research and its implementation stages. They were also assured about the confidentiality of their information and were free to leave the study whenever they wished, and if desired, the research results would be available to them.

Funding

This research did not receive any grant from funding agencies in the public, commercial, or non-profit sectors.

Authors' contributions

All authors equally contributed to preparing this article.

\section{Conflicts of interest}

The authors declared no conflict of interest. 


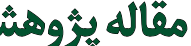

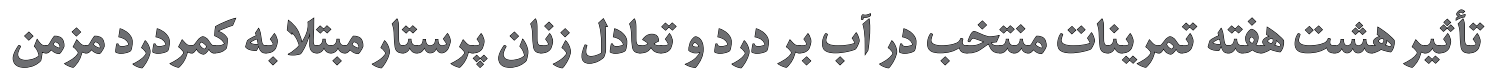

\author{
"هوار ايزدى' ته، نرمين غنىزاده حصار” (1) \\ 1. كروه آسيب شناسى ورزشى وحركات اصلاحى، دانشكده تربيت بدنى، واحد اروميه، دانشكاه آزاد اسلامى، اروميه، ايران.

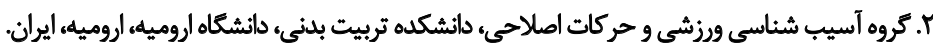

هدف هدف از مطالعه حاضر، بررسى تأثير هشت هفته تمرينات منتخب در آب بر دردو تعادل زنان يرستار مبتلا به كمردرد مزمن است.

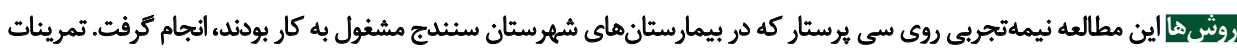

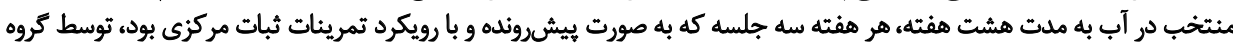

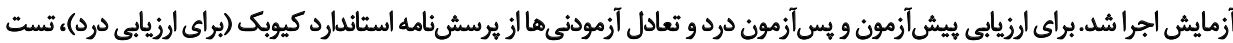

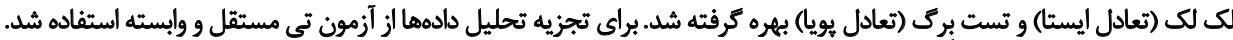

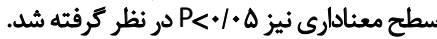

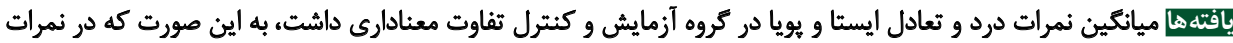

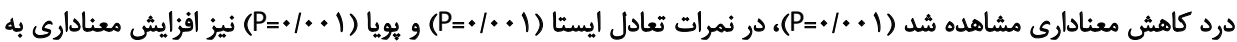

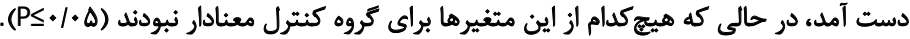

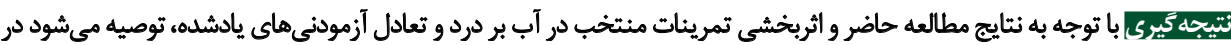

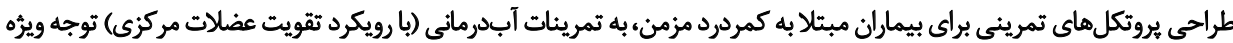

محققين معتقدند اكر جه در • •

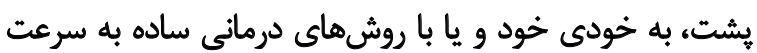

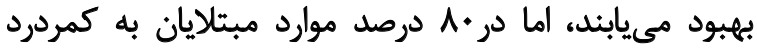

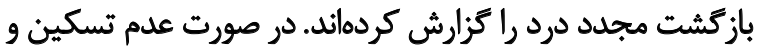

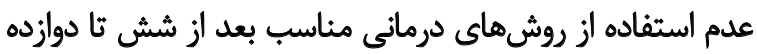

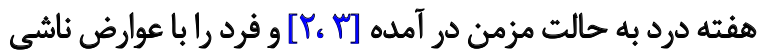
از دردهاى مزمن رو بهرو مي كند.

بازتشت دردهاى ناحيه كمر با اختلالات عملكردى، محدود

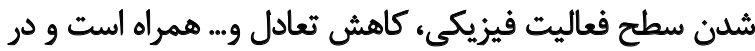

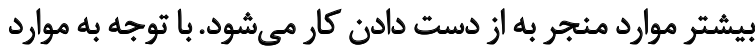

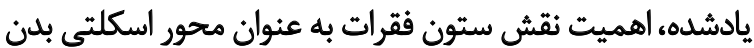

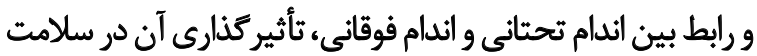
افراد و وضعيت بدنى مطلوب بديهيى به نظر مىرسداني

ستون فقرات به عنوان محور حركتى بدن در اعمال و

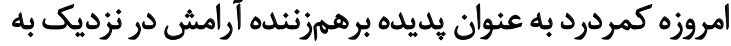

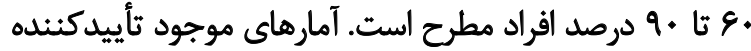

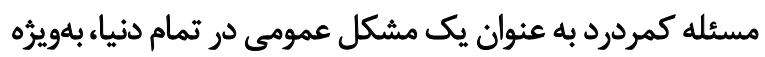

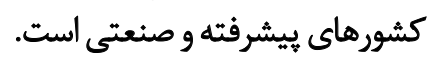

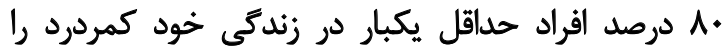

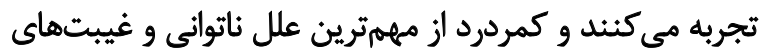

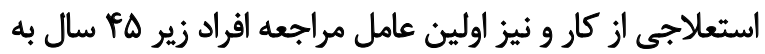

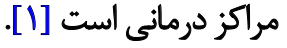
اكر ها دلايل زيادى از يك كشيدكى ساده عضلانى تا يك توده

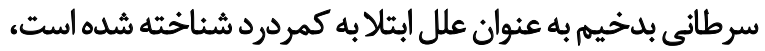

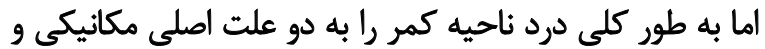
دردهاى انتشارى نسبت دادهاند. 
هنكامى كه ثبات ناحيه كمرى لكنى افزرايش ييدا مي كند،اغلب

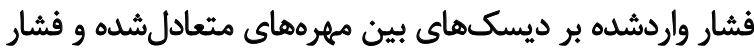

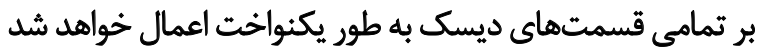

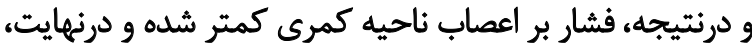

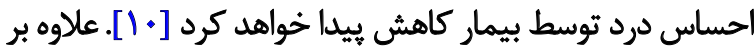

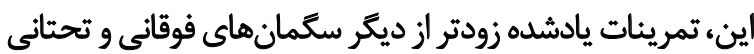

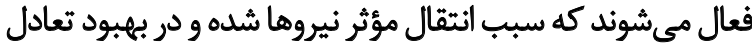
نقش بسرايع را ايفا مى كنيند.

در اين ميان، تمرين در آب، به طور مكرر در توانبخشى

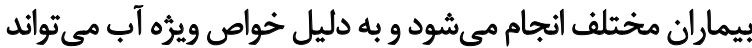

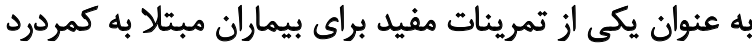

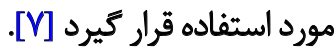
آبدرمانى به سبب كاهش فشار داخل ديسك، اندازه سوراخ

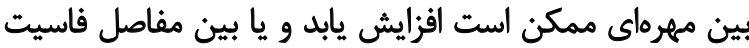

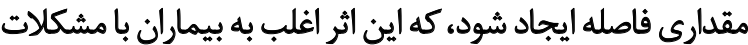

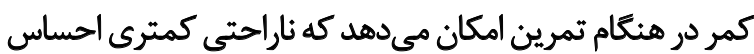

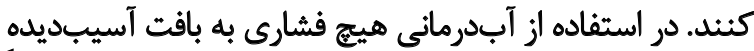

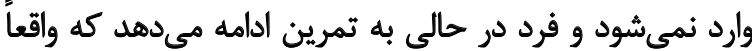

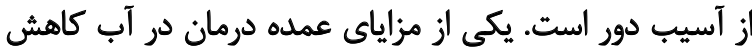

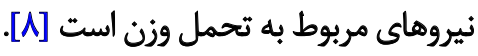

بنابراين با توجه به شيوع بيمارى كمردرد در يرستاران (حدود

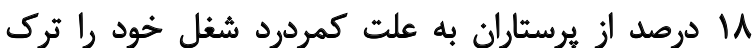

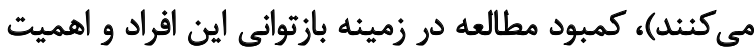

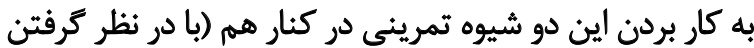

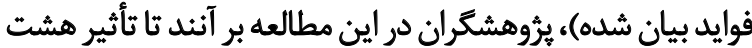

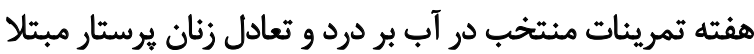
به كمردرد مزمن غير اختصاصى بسنجند.

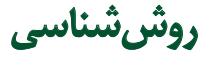
مطالعه حاضر نيمهتجربى بوده كه با شركت دوطلبانه سي

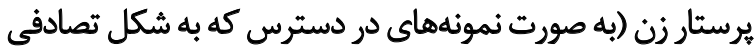

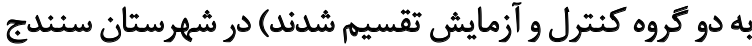

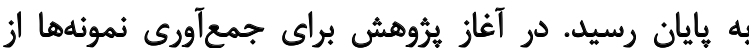

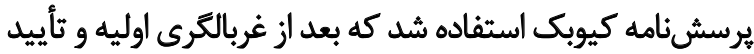

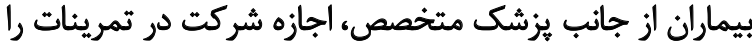

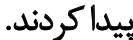

بيش از شروع يثوهش، براى افراد شرح داده شد كه در هر زمان

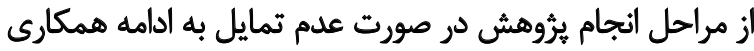

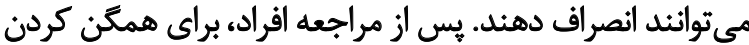

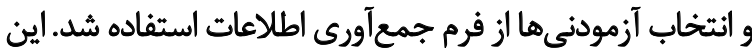

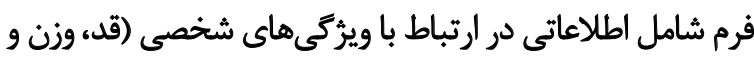
سن) و سابقه آسيب در ناحيه ستون فقرات كمرى بوديات
فعاليتهاى مختلف همواره در معرض آسيب و ناهنجارى قرار

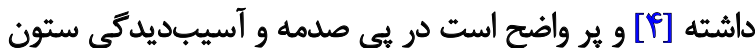

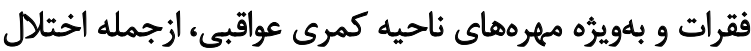

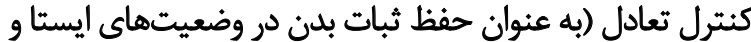

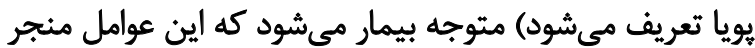

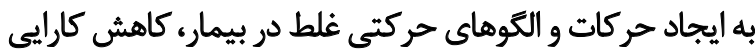

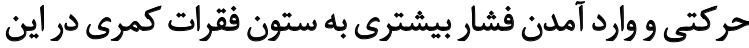

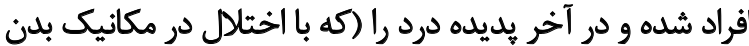

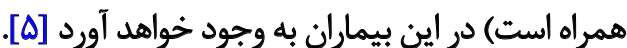
ز زرفى كمردرد به عنوان يك بيمارى مرتبط با كار، از

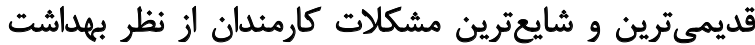

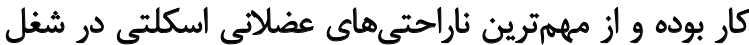

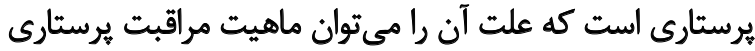
برشمرد [ه].

علاوه بر فشارهاى فيزيكى زياد در اين حرفه مانند جابهجايى

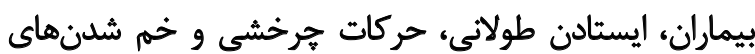

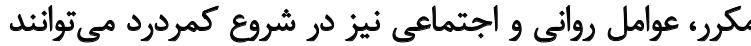

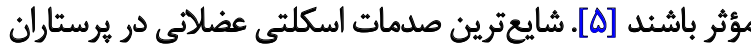

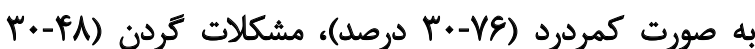
درصد) و شانه (Tr-هr

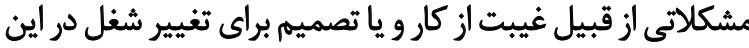

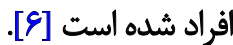

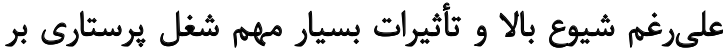

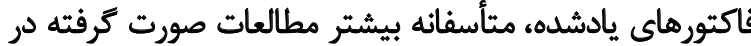

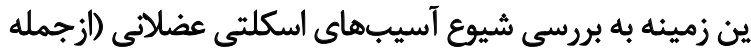

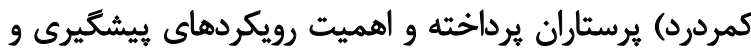

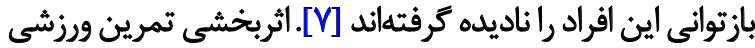

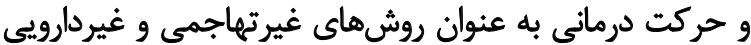

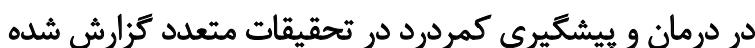

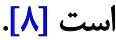

مطالعات انجامشده، نشان داده كه انجام تمرينات ورزشي به بإيه

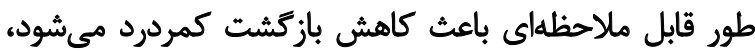

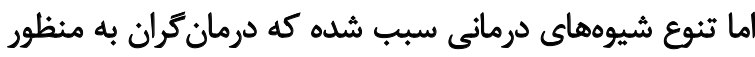

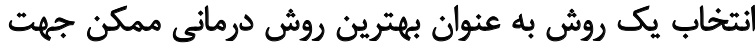

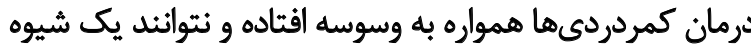

تمرينى رابه عنوان بهترين تمرين جهت درداره درمان معرفى كنيند. تمرينات ثباتدهنده عملكردى و آبدرمانى در دها آنيا

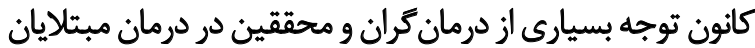

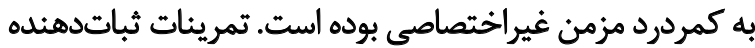

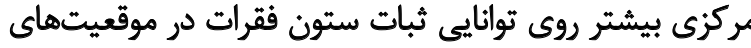

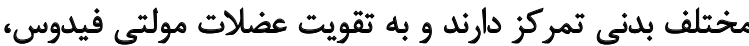

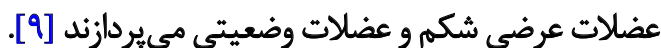


جدول ا. تمرينات ثبات مركزى در آب

\begin{tabular}{|c|c|c|c|c|c|c|}
\hline استراحت بين هر حركت & زمان تمرين & ت تكرار & سبت & جلسه & نوع تمرين & هفته \\
\hline إثانيه & 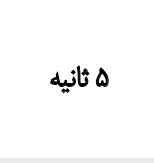 & 8 & $f$ & سول & كشش عضلات همسترينك & اول \\
\hline و بثاثيه & هـ ثانيه & $\&$ & f & عود & كشش عضله كلوتثوس ماكزيموس & دوم \\
\hline ع ثانيه & ا ثاثيه & 8 & $r$ & دوم & كشش عضلات تنه (خلفى) & سوم \\
\hline . & ا ثاثيه & $\varepsilon$ & f & سوم & متمايل كردن لكن & جهارم \\
\hline •ع ثائيه & ها ثائيه & 8 & $r$ & سول & جمع كردن لكن & ينجمم \\
\hline (ع ثائيه & هائيه & 8 & f & سوم & خم كردن تنه با نوار الاستيك & sin \\
\hline و ثائيه & إثانيه & 8 & $r$ & سو & به سمت زانو آوردن ققسه سيئه & هفتم \\
\hline . ع ثائيه. & • ب ثائيه & 8 & r & سوم & حفظ تيلت لكن در وضعيت ايستاده دور كردن باها در & هشتم \\
\hline
\end{tabular}

مجله بيومكانيك ورنث

يس از انجام هشت هفته تمرينات تقويتى عضلات ثبات دهنيده

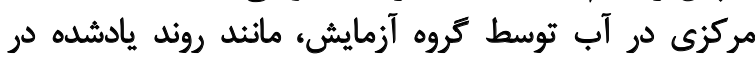

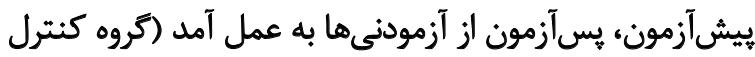

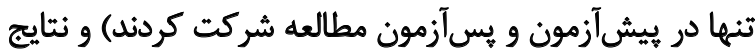

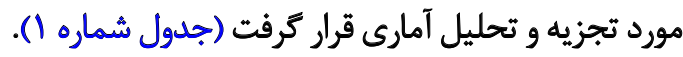
نحوه ارزيابى ميزان درد كمر: ميزان درد ناشى از كمردرد توسط

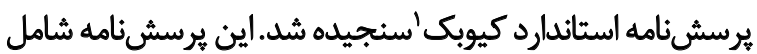

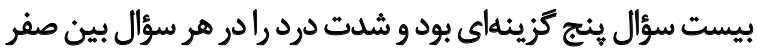

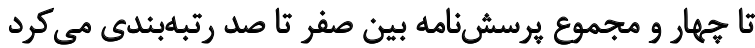

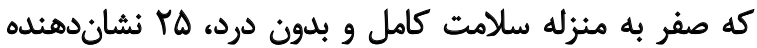

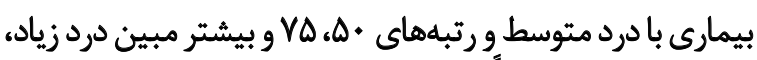
درد خيلى زياد و كاملا حاد بود.

آزمودنىها اين يرسشنامه را يكبار قبل از اجراى يروتكل

1. Quebec Standard Questionnaire
از اطلاعات موجود در فرم با توجه به معيار هاي ورود تعيينشده

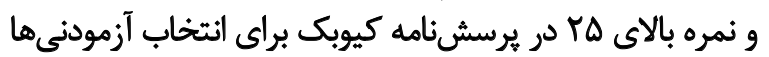

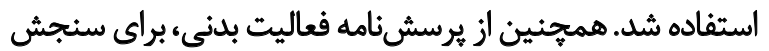

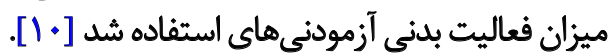

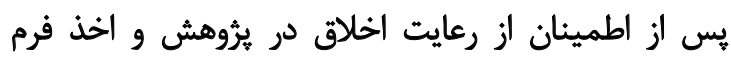

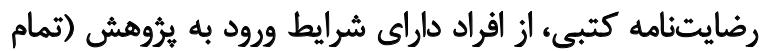

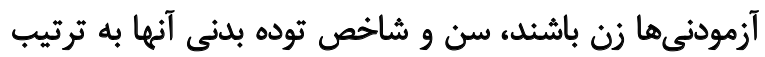

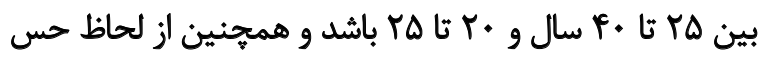

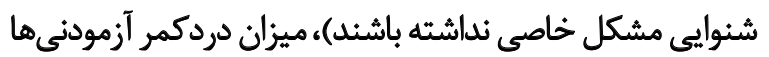

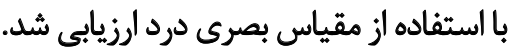

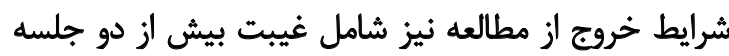

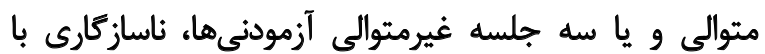

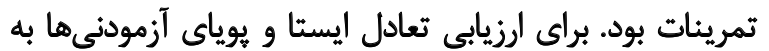

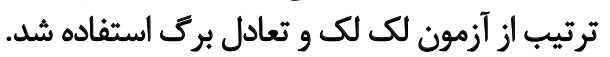


جدول r. مشخصات جمعيتشناختى آزمودنىهاى در دو كروه كنترل و آزمايش

\begin{tabular}{|c|c|c|}
\hline \multicolumn{2}{|c|}{ ميانكين||نحراف استاندارد } & \multirow{2}{*}{ متغير } \\
\hline تروه تجريى (تعداد ه ا نفر) & تروه كتترل (تعداد: 0 انفر) & \\
\hline$\Pi \pm \Psi / \Lambda$ & $r \Delta \pm \psi / 1$ & سن (سال) \\
\hline $199 \pm r / q$ & $\mid \varepsilon A \pm r / 9$ & قد (سانتىمتر) \\
\hline$n \pm r / r$ & $V \cdot \pm 1 / f$ & وزن (كيلوكرم) \\
\hline
\end{tabular}

مجله بيومكانيك وزنشـ

دربركيرنده فعاليتهاى روزانه، ازجمله نشستن، ايستادن و انتقال

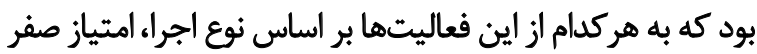

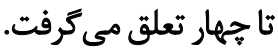

بيشترين امتياز وها لأ)، (نشاندهنده كمترين ريسك افتادن)

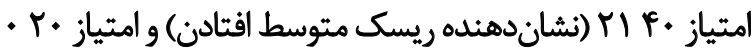

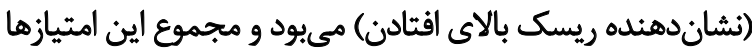

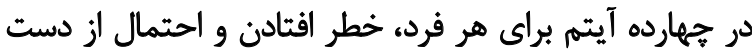

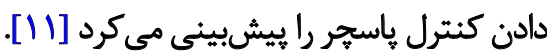
يس از انجام معاينات، آزمونهاي اوليه و شكل كيرى دو گروه،

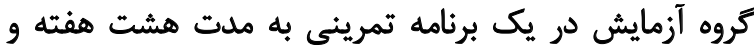

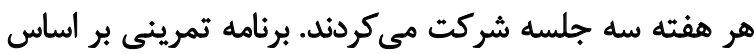

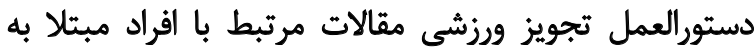

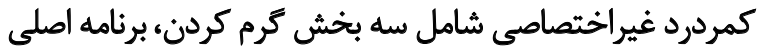
تمرين و سرد كردن بود. مدت زمان برنامه اصلى تمرين بر اساس تعداد تكرارهاي هر

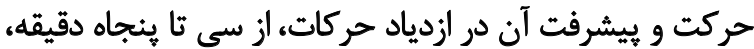

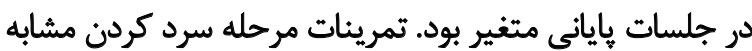

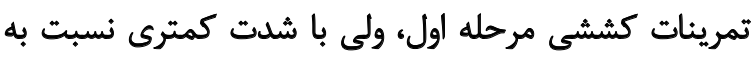

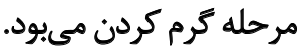

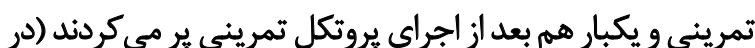

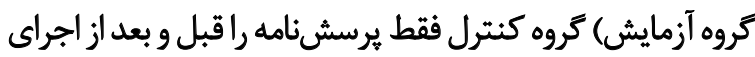

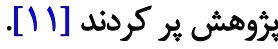

$$
\text { اندازهتيرى تعادل ايستا و بويا }
$$

تعادل ايستا: براى ارزيابى تعادل ايستا از آزمون لك لك استفاده

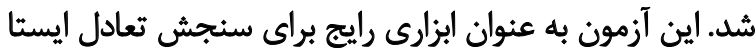

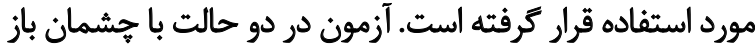

$$
\text { و بسته به شرح زير انجام كرفت. }
$$

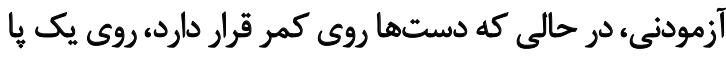

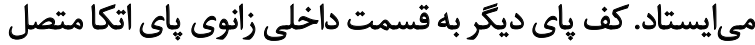

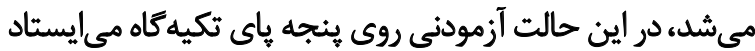

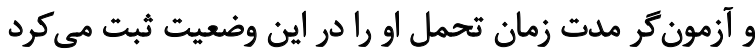

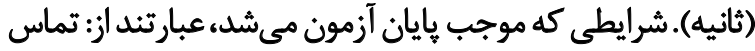

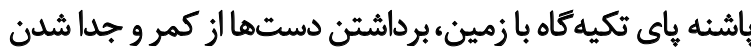

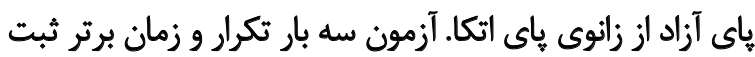

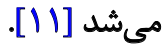

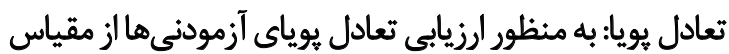

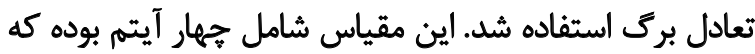

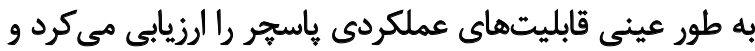

\begin{tabular}{|c|c|c|c|c|c|}
\hline \multirow{2}{*}{ P درون Sروهى P P } & \multirow{2}{*}{ مقدار } & \multicolumn{2}{|c|}{ ميانكين+|نحراف استاندارد } & \multirow{2}{*}{ 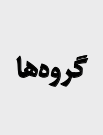 } & \multirow{2}{*}{ متغير } \\
\hline & & يس آزمون & ي يشي آزمون & & \\
\hline.$/ 410$ &.$- / 4 r 1$ & $r q / q \Psi \pm R / r T$ & $r q / r \cdot \pm I r / r q$ & كتنرل & شلت درد \\
\hline $.1 . .1$ & NMI & $\mid F / T \Delta \pm N N$ & $r \cdot / r \Delta \pm \mid F / \cdot V$ & أزمايش & شدت درد \\
\hline $1 / \lambda^{+}$ & $-1 / 18$ & 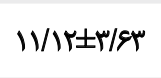 & $1+/ N \pm r / \Delta r$ & كنترل & تعادل ايستا (ثانيه) \\
\hline $.1 . .1$ & $-|\varepsilon /| r$ & $r g / q) \pm \varepsilon / P r$ & $1 . / \mid V \pm f(\Delta)$ & آزمايش & تعادل ايستا (ثائيه) \\
\hline .118 & $-1 / 48$ & $\pi / n^{*} \pm r / r \Lambda$ & $\pi r / r \cdot \pm r / T r$ & كتثرل & تعادل يويا برى (نمره) \\
\hline $.1 . .1$ & $-1 \Delta / 2 \Delta$ & $\Gamma \Delta / \Delta T \pm r / W$ & $M r / c \cdot \pm r / M I$ & أزمايش & تعادل يويا برى (ثمره) \\
\hline
\end{tabular}

جدول "ا. بررسى نمرات شدت درد، تعادل ايستاو يويا در مقايسه تى همبسته در ييشآزمون و يسآزمون كروه آزمايش و كنترل

مجله بيومكانيك ولشث 
جدول F. تتايج آزمون تى مستقل براى مقايسه نمرات شدت درد، تعادل ايستاو يويا در بيشآزمون و يسآزمون در كروه كنترل و آزمايش

\begin{tabular}{|c|c|c|c|c|c|c|c|}
\hline $\mathbf{P}$ & $\mathbf{t}$ & اختّاف ميانكين بس أزمون & $\mathbf{P}$ & $\mathbf{t}$ & اختلاف ميانكين ييش آزمون & ت تمروها & مثغير \\
\hline $.1 .+1$ & $V / \Delta q$ & 10/98 &.$/ 11$ & VIST & $V \cdot \Delta$ & كتجرل & شدت درد \\
\hline $.1 .+1$ & זוN- & -181.9 & . NE &.$/ 9 \Delta$ & $\cdot / M$ & كتبرل & 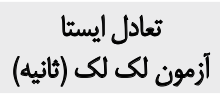 \\
\hline $.1 . .1$ & $-q /{ }^{2} \Delta$ & $-11 / \% a$ & $. / \Delta \mid$ & .198 & $\cdot / A$. & كتربيى & تعادل يويا برى (نمره) \\
\hline
\end{tabular}

مجله بيومكانيك وزنث

در ييشآزمون گروه كنترل و آزمايش در متغيرهاى شدت درد،

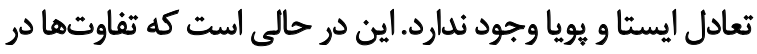

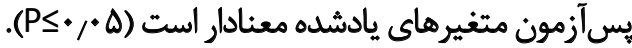
A

هدف از يُوهش حاضر، بررسى اثر هشت هفته تمرينات

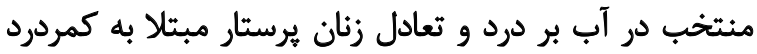

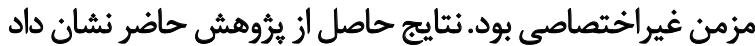

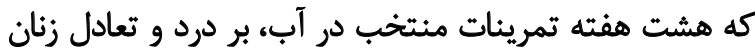

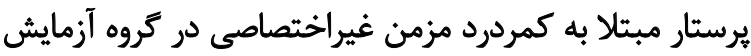
تأثير معنادارى داشته است.

اين در حالى است كه نتايج بهدست آمده براى كروه كنترل

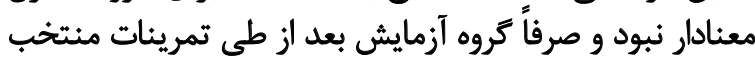

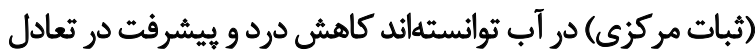

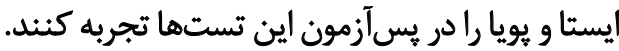

نتايج بهدست آمده در رابطه با درد و تعادل ايستاو يويا با بنتايج

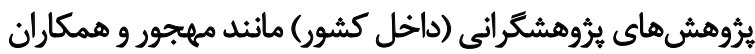

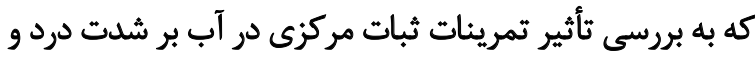

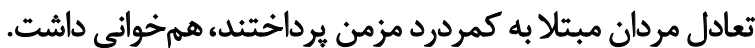

همجنين با نتايج ثرؤهش نظرزاده و همكاران، نراد رومزى و و

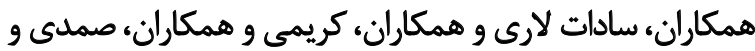

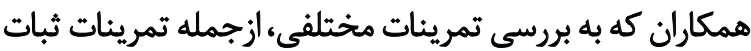

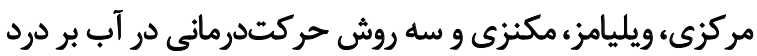

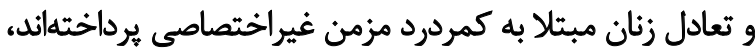

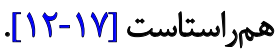

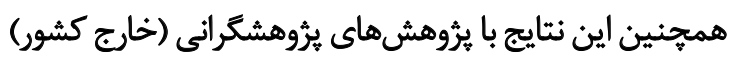

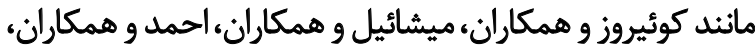

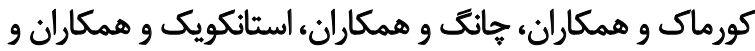

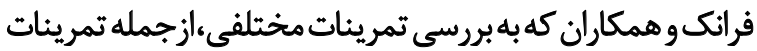

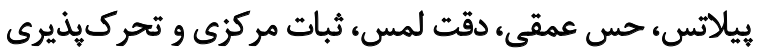

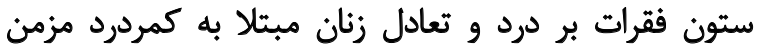

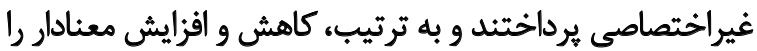

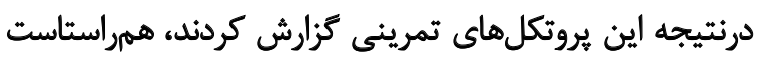

با توجه به توصيههاى مختلف درخصوص زمان نتخهدارى

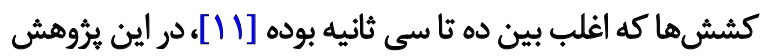

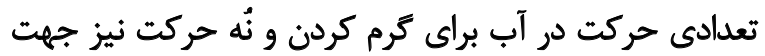

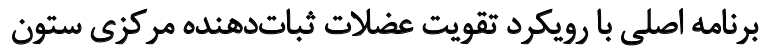

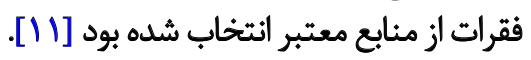

روش تجزيه و تحليل: در اين بروهش از آز آزمون نرماليته

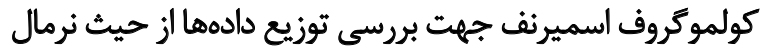

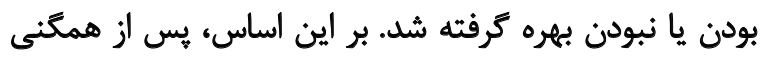

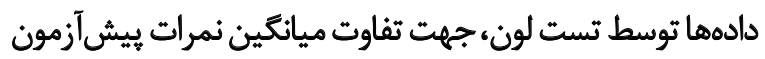

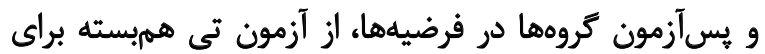

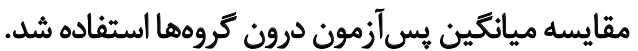

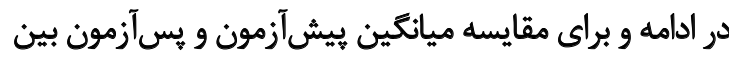

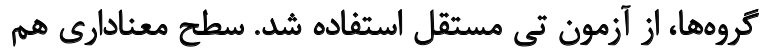

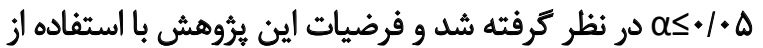

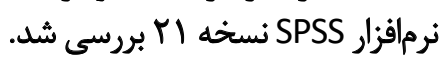

\section{نتايج}

مشخصات جمعيتشناختى آزمودنىهاى كروه كنترل و ورائ

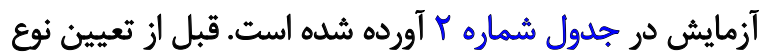

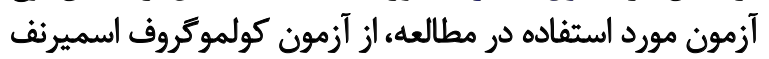
جهت بررسى نرمال بودن متغيرها استفاده شد. در جدول شماره آ، از آزمون تى همبسته براي بررسى نمرات

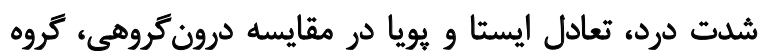
كنترل و آزمايش استفاده شده است. با توجه به جدول بالا، نتايج مربوط به شدت درد، تعادل ايستاو

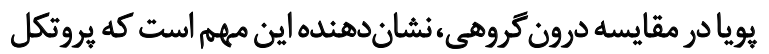

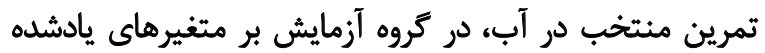

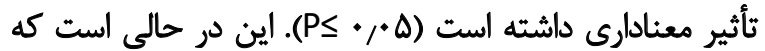

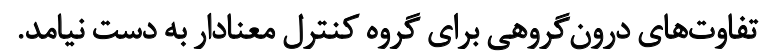

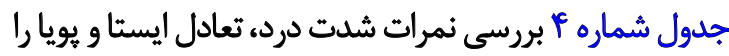

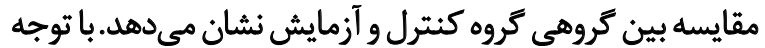

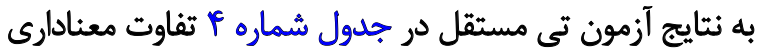


بهبود عملكرد در زنان مبتلا به كمردرد مزمن داشته باشد. با توجه به نتايج بلددستآمده در اين تحقيق، به نظر مىرسد

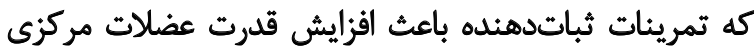

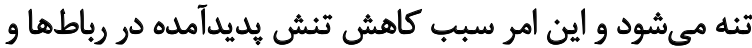

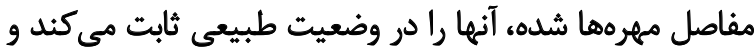

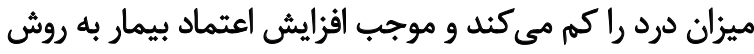

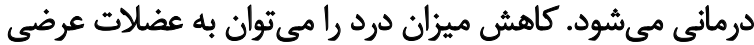

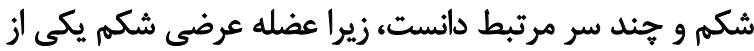

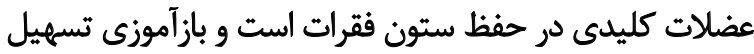

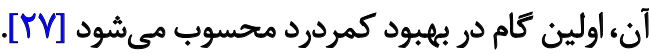

در رابطه با مكانيزم احتمالى بهبود تعادل بيماران زن مبتمانلا

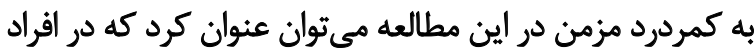

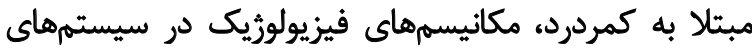

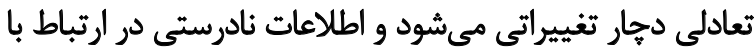

وضعيث فضايى بدن به ساقه مغز ارسال مى شيود [ · []. اين اطلاعات باعث ارسال دستورات حركتى نامناسب و متعاقب

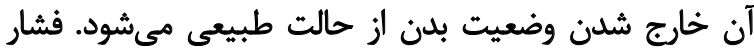

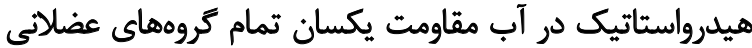

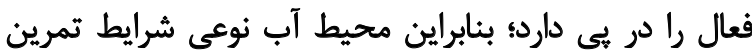

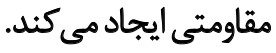

جمكالى بيشتر آب نسبت به هوا كمك مي كند تا نيروى مقاوم

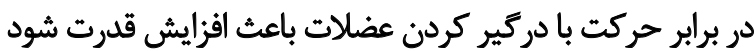

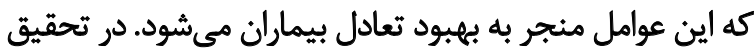

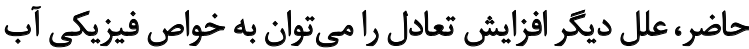

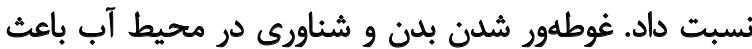

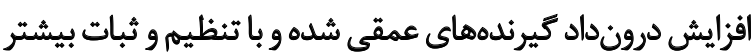
بدن منجر به بهبود تعادل مي مئدود.

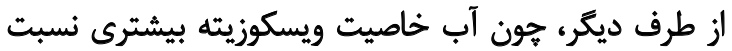

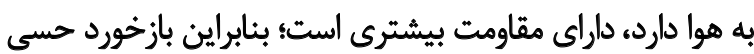

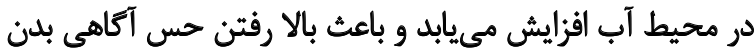

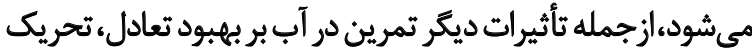

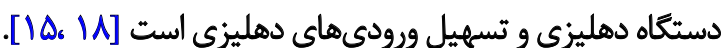

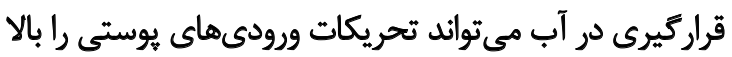

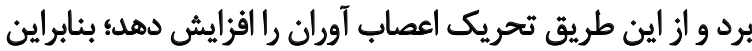

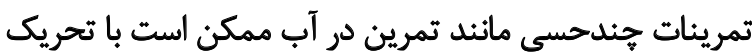

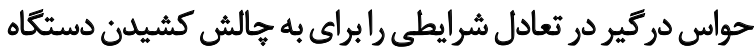

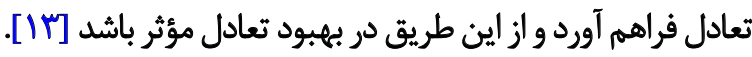

از طرفى، از آنجا كه تمرينات منتخب در آب اين يثروهش باديات

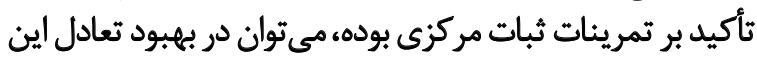

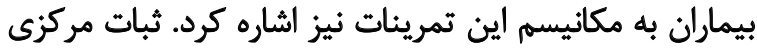

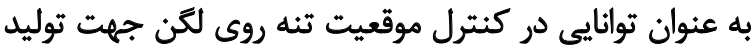

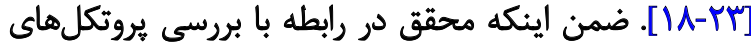

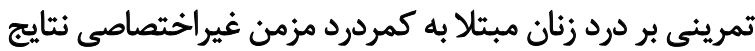
متناقضى نيافته است.

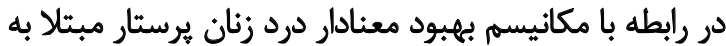

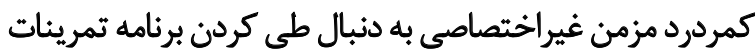

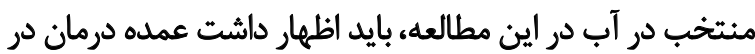

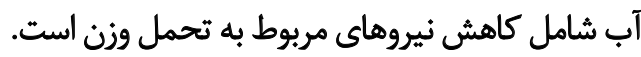

بيمارانى كه در آب تمرين مى كنند، احساس سبك بودن و

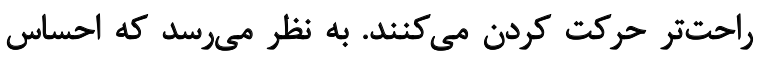

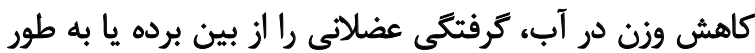

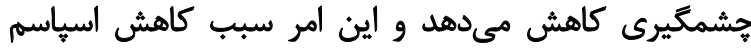

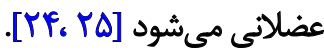

بكر بر اين اعتقاد است كه تمريناتي كه در آنها وزن بلدن تحمل

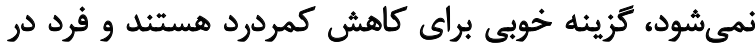

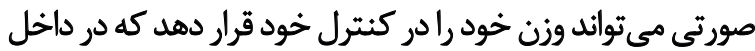

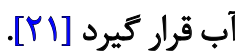

شنا كردن، دراز كشيدن و انجام تمرينات در آب يكى الز بهترين

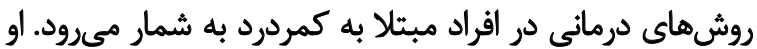

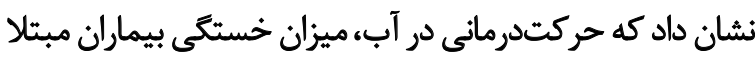

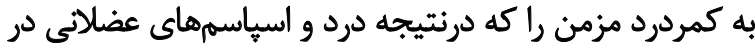

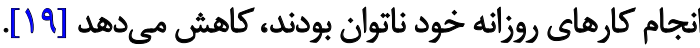
از طرفى، در اين مطالعه از تمرينات ثبات مركزى به عندوان

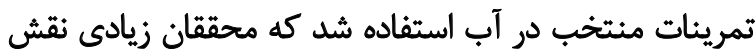

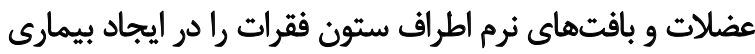

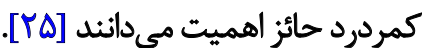

اساس استدلال آنها اين است كه ضعف عضلات جهت

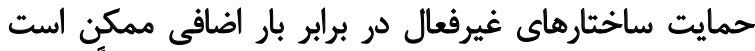

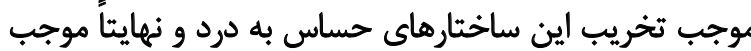

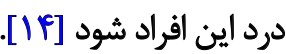

مطالعات قبلى به اين نتيجه رسيدند كه اختلال و ضعف در

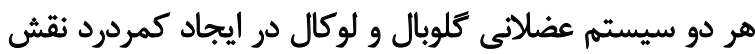

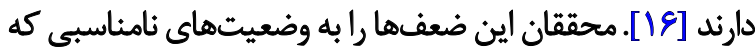

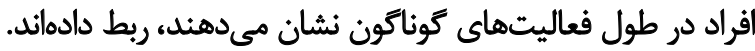

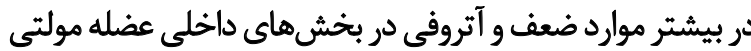

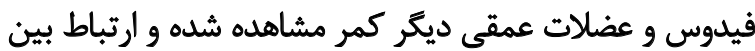

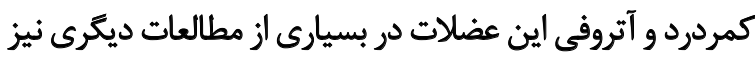

اثبات شده است [Y [Y].

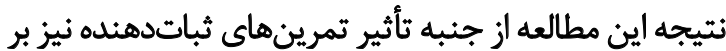

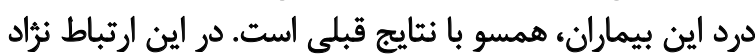

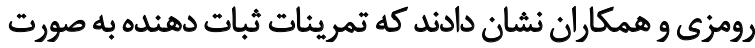

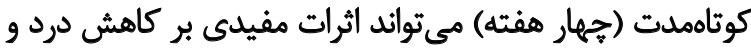




$$
\text { مشاركت نويسند }
$$

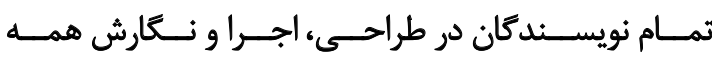

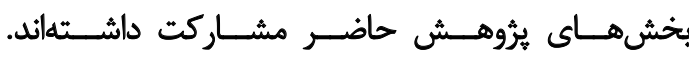

$$
\text { تعارض مناقع }
$$

بنابر اظهار نويسندكان اين مقاله تعارض منافع ندارد.
و انتقال مؤثر نيرو به بخشهاي انتهايى بدن در طول فعاليتها

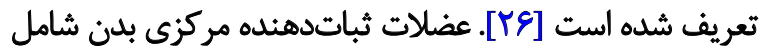

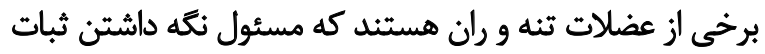

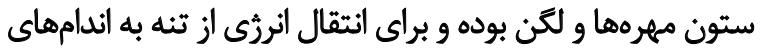

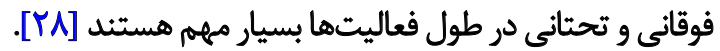
عملكرد و هماهنكى مناسب عضلات اين ناحيه از بدن جهت

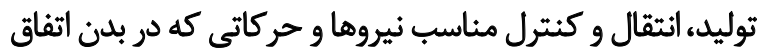

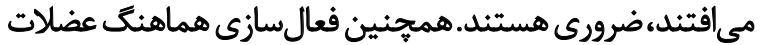

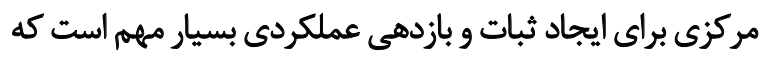

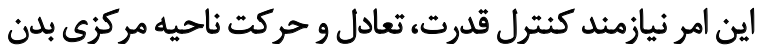

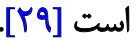

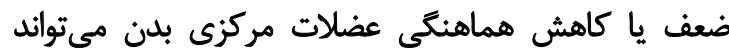

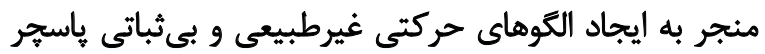

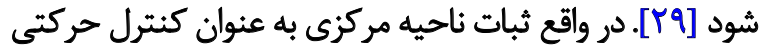
و ظرفيت عضلانى ناحيه مركزى، براى حفظ ثبات ثبات اين ناحيه

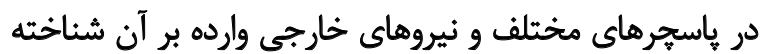

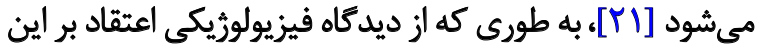

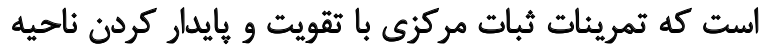

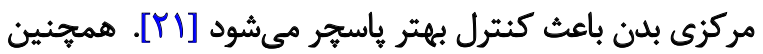

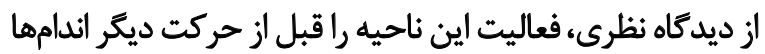

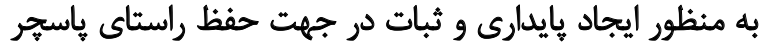

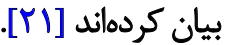

\section{نتيجهيَيرىنهايى}

با توجه به نتايج معنادار مطالعه حاضر و اثربخشى احتمالى تمرينات منتخب در آب بر درد و تعادل آزمودنى هائ يادئ يادشدهاء

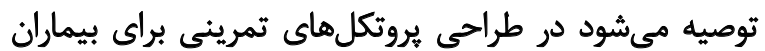

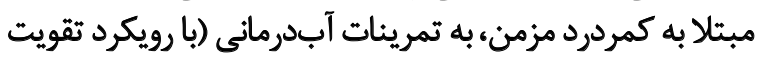
عضلات مركزى) توجه ويثه شود.

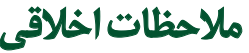

$$
\text { ييروى از اصول اخلاق بثروهش }
$$

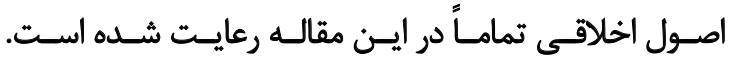

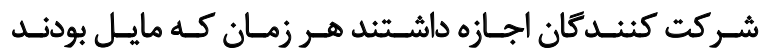

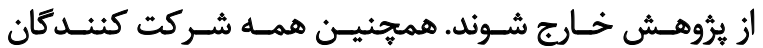

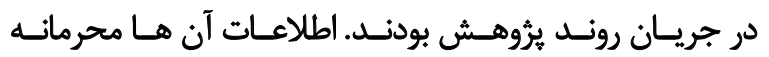

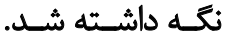

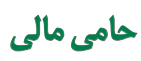

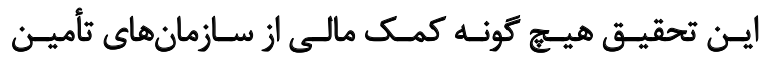

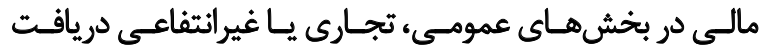

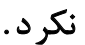




\section{Reference}

[1] Ramazani Badr F, Nikbakht A, Mohammadpour A. Low-back pain prevalence and its risk factors in nurses. Iran J Nursing Res. 2006; 1(2):37-42. http://ijnr.ir/browse.php?a_id=30\&sid=1\&slc_lang=en

[2] Waddell G, Burton AK. Occupational health guidelines for the management of low back pain. Occup Med. 2001; 51(2):124-35. [DOI:10.1093/occmed/51.2.124] [PMID]

[3] Agilinejad M, Mostafaei M. [Occupational medicine and professional diseases (Persian)]. Tehran: Arjmand Pub. https://www.gisoom. com/book/1362648/\%DA\%A9\%D88C/

[4] Tinubu BMS, Mbada CE, Oyeyemi AL, Fabunmi AA. Work-related musculoskeletal disorders among nurses in Ibadan, south-west $\mathrm{Ni}$ geria: A cross-sectional survey. BMC Musculoskelet Disord. 2010; 11:12. [DOI:10.1186/1471-2474-11-12] [PMID] [PMCID]

[5] Mohseni-Bandpei MA, Fakhri M, Ahmad-Shirvani M, Bagheri Nessami M, Khalilian AR. [Epidemiological survey of low back pain among nurses (Persian)]. J Babol Uni Med Sci. 2005; 7(26):35-40. http:// jbums.org/browse.php?a_id=2635\&sid=1\&slc_lang=fa

[6] Hoy D, Brooks P, Blyth F, Buchbinder R. The epidemiology of low back pain. Best Pract Res Clin Rheumatol. 2010; 24(6):769-81. [DOI:10.1016/j.berh.2010.10.002] [PMID]

[7] Ferreira ML, Ferreira PH, Latimer J, Herbert R, Maher C. Dose spinal manipulative therapy help people with chronic low back pain? Australian J Plant Physiol. 2002; 48(4):277-84. [DOI:10.1016/S00049514(14)60167-7]

[8] Mostafazade F, Rostamzade M, Mashofi M, Afzalifard H. [Assessing quality of life in low back pain admitted in Ardebil Physiography Center 2004 (Persian)]. Aflak. 2005; 2(2-3). https://eprints.arums.ac.ir/631/

[9] Harreby M, Kjer J, Hesselsoe G, Neergaard K. Epidemiological aspects and risk factors for low back pain in 38-year-old men and women: A 25-year prospective cohort study of 640 school children. Eur Spine J. 1996; 5(5):312-8. [DOI:10.1007/BF00304346] [PMID]

[10] Detmar SB, Aaronson NK. Quality of life assessment in daily clinical oncology practice: A feasibility study. Eur J Cancer. 1998; 34(8):1181-6. [DOI:10.1016/S0959-8049(98)00018-5]

[11] Mousavi SJ, Parnianpour M, Mehdian $\mathrm{H}$, Montazeri A, Mobini B. The Oswestry Disability Index, the Roland-Morris Disability Questionnaire, and the Quebec Back Pain Disability Scale: Translation and validation studies of the Iranian versions. Spine. 2006; 31(14): 454-9. [DOI:10.1097/01.brs.0000222141.61424.f7] [PMID]

[12] Oleson M. Subjectively perceived quality of life. Image J Nurs Sch. 1990; 22(3):187-90. [DOI:10.1111/j.1547-5069.1990.tb00205.x] [PMID]

[13] Bagheri S, Sarmadi A, Dadashi L. Learning effects of the biodex balance system during assessment of postural task with in test-retest measurements. Koomesh. 2012; 13(3):354-63. http://koomeshjournal.semums.ac.ir/article-1-1455-en.html

[14] Carpes FP, Reinehr FB, Mota CB. Effects of a program for trunk strength and stability on pain, low back and pelvis kinematics, and body balance: A pilot study. J Bodyw Mov Ther. 2008; 12(1):22-30. [DOI:10.1016/j.jbmt.2007.05.001] [PMID]

[15] Resende SM, Rassi CM, Viana FP. Effects of hydrotherapy in balance and prevention of falls among elderly women. Rev Bras Fisioter 2008; 12(1):57-63. [DOI:10.1590/S1413-35552008000100011]

[16] Roth A, Miller MG, Ricard MD, Ritenour D, Chapman BL. Comparisons of static and dynamic balance following training in aquatic and land environments. J Sport Rehabil. 2006; 15(4):299-311. [DOI:10.1123/jsr.15.4.299]
[17] Kawasaki JM. The effects of aquatic exercise on balance outcomes in individuals with Parkinson's disease. Med Sci Sports Exercise 2009; 41(5):102. [DOI:10.1249/01.MSS.0000354870.87591.d9]

[18] Gillespie LD, Robertson MC, Gillespie WJ, Lamb SE, Gates S, Cumming RG, et al. Interventions for preventing falls in older people living in the community. Cochrane Database Syst Rev. 2012; 2012(9):CD007146. [DOI:10.1002/14651858.CD007146.pub3] [PMID] [PMCID]

[19] Viliani T, Pasquetti P, Magnolfi S, Lunardelli ML, Giorgi C, Serra P, et al. Effects of physical training on straightening-up processes in patients with Parkinson's disease. Disabil Rehabil. 1999; 21(2):68-73. [DOI:10.1080/096382899297990] [PMID]

[20] Kopec JA, Esdaile JM, Abrahamowicz M, Abenhaim L, Wood Dauphinee S, Lamping DL, et al. The Quebec Back Pain Disability Scale. Measurement properties. Spine. 1995; 20(3):341-52. [DOI:10.1097/00007632-199502000-00016] [PMID]

[21] Waller B, Lambeck J, Daly D. Therapeutic aquatic exercise in the treatment of low back pain: A systematic review. Clin Rehabil. 2009; 23(1):3-14. [DOI:10.1177/0269215508097856] [PMID]

[22] Dundar U, Solak O, Yigit I, Evcik D, Kavuncu V. Clinical effectiveness of aquatic exercise to treat chronic low back pain: A randomized controlled trial. Spine. 2009; 34(14):1436-40. [DOI:10.1097/ BRS.0b013e3181a79618] [PMID]

[23] Sedaghati N, Hematfar A, Behpour N. [The effect of a selected spinal core-muscle stabilization training in water on pain intensity and lumbar lordosis (Persian)]. Feyz. 2013; 17(3):267-74. https://www. sid.ir/fa/journal/ViewPaper.aspx?id=216136

[24] Lee JH, Ooi Y, Nakamura K. Measurement of muscle strength of the trunk and the lower extremities in subjects with history of low back pain. Spine. 1995; 20(18):1994-6. [DOI:10.1097/00007632199509150-00006] [PMID]

[25] Rutledge E, Silvers WM, Browder K. Metabolic-cost comparison of submaximal land and aquatic treadmill exercise. Int J Aquat Res Educ. 2007; 1(2):118-33. [DOI:10.25035/ijare.01.02.04]

[26] Hernandez-Reif M, Diego M, Field T. Preterm infants show reduced stress behaviors and activity after 5 days of massage therapy. Infant Behav Dev. 2007; 30(4):557-61. [DOI:10.1016/j.infbeh.2007.04.002] [PMID] [PMCID]

[27] Ruhe A, Fejer R, Walker B. Center of pressure excursion as a measure of balance performance in patients with non-specific low back pain compared to healthy controls: A systematic review of the literature. Eur Spine J. 2011; 20(3):358-68. [DOI:10.1007/s00586-0101543-2] [PMID] [PMCID]

[28] Arendt-Nielsen L, Graven-Nielsen T. Muscle pain: Sensory implications and interaction with motor control. Clin J Pain. 2008; 24(4):291-8. [DOI:10.1097/AJP.0b013e31815b608f] [PMID]

[29] Moseley GL, Hodges PW. Are the changes in postural control associated with low back pain caused by pain interference? Clin J Pain. 2005; 21(4):323-9. [DOI:10.1097/01.ajp.0000131414.84596.99] [PMID] 
This Page Intentionally Left Blank 